\title{
SUPERMETRICS ON SUPERMANIFOLDS
}

\author{
G.SARDANASHVILY \\ Department of Theoretical Physics, Moscow State University, 117234, Moscow, Russia
}

\begin{abstract}
By virtue of the well-known theorem, a structure Lie group $K$ of a principal bundle $P \rightarrow X$ is reducible to its closed subgroup $H$ iff there exists a global section of the quotient bundle $P / K \rightarrow X$. In gauge theory, such sections are treated as Higgs fields, exemplified by pseudoRiemannian metrics on a base manifold $X$. Under some conditions, this theorem is extended to principal superbundles in the category of $G$-supermanifolds. Given a $G$-supermanifold $M$ and a graded frame superbundle over $M$ with a structure general linear supergroup, a reduction of this structure supergroup to an orthgonal-symplectic supersubgroup is associated to a supermetric on a $G$-supermanifold $M$.
\end{abstract}

\section{Introduction}

Let $\pi: P \rightarrow X$ be a principal smooth bundle with a structure Lie group $K$. One says that its structure group $K$ is reducible to a closed (consequently, Lie) subgroup $H$ of $K$, if there exists an $H$-principal subbundle of $P$. The following well-known theorem holds [1].

Theorem 1. There is one-to-one correspondence between the reduced $H$-principal subbundles $P^{h}$ of $P$ and the global sections $h$ of the quotient bundle $P / H \rightarrow X$.

In gauge theory on a principal bundle $P \rightarrow X$, these sections $h$ are regarded as classical Higgs fields [2, 3] In particular, let $P=L X$ be the $G L(n, \mathbb{R})$-principal bundle of linear frames in the tangent bundle $T X$ of $X(\mathrm{n}=\operatorname{dim} \mathrm{X})$. If $H=O(k, n-k)$, then a global section of the quotient bundle $L X / O(k, n-k)$ is a pseudo-Riemannian metric on $X$. For instance, this is the case of a gravitational field in gauge gravitation theory $[4,5]$.

SUSY gauge theory including supergravity is mainly developed as a Yang-Mills type theory with spontaneous breaking of supersymmetries $[6,7,8]$. However, supergravity introduced in SUSY gauge theory has no geometric feature as a supermetric [9, 10]. Therefore, our goal here is to extend Theorem 1 to principal superbundles.

It should be emphasized that there are different notions of a supermanifold, Lie supergroup and superbundle. Let us mention a definition of a super Lie group as a HarishChandra pair of a Lie group and a super Lie algebra [10, 11]. We are not concerned with graded manifolds, graded Lie algebras and principal bundles $[12,13,14]$. A problem is that the tangent bundle of a graded manifold fails to be a graded manifold, but it is locally a 
Ne'eman-Quillen superbundle [15] (see [16, 17, 18] for different definitions of a supermetric on graded manifolds). It should be also emphasized that graded manifolds are not supermanifolds in a strict sense. Both graded manifolds and supermanifolds are described in terms of sheaves of graded commutative algebras [19]. However, graded manifolds are characterized by sheaves on smooth manifolds, while supermanifolds are constructed by gluing of sheaves on supervector spaces.

We here restrict our consideration to supermanifolds over Grassmann algebras $\Lambda$ of finite rank. This is the case of smooth $\left(\mathrm{GH}^{\infty_{-}}, \mathrm{H}^{\infty_{-}}, G^{\infty_{-}}\right)$supermanifolds and $G$-supermanifolds [19]. By analogy with manifolds, smooth supermanifolds are constructed by gluing of open subsets of supervector spaces $B^{n, m}$ endowed with the Euclidean topology. If a supervector space $B^{n, m}$ is provided with the non-Hausdorff DeWitt topology and a smooth or $G$-supermanifold admits an appropriate atlas, it is a DeWitt supermanifold.

In a general setting, one considers supermanifolds over the so called Arens-Michael algebras of Grassmann origin. They are $R$ - and $R^{\infty}$-supermanifolds which obey a certain set of axioms in order to be most suitable for supergeometry [20,21, 22]. In the case of a finite Grassmann algebra, the category of $R^{\infty}$-supermanifolds is equivalent to the category of $G$ supermanifolds. In comparison with smooth supermanifolds, $G$-supermanifolds have some important advantages from the differential geometric viewpoint [19]. Firstly, derivations of the structure sheaf of a $G$-supermanifold $\widehat{M}$ constitute a locally free sheaf, which is the structure sheaf of some $G$-superbundle $T \widehat{M}$ regarded as a tangent superbundle of $\widehat{M}$. Secondly, the category of $G$-supervector bundles is equivalent to a certain category of locally free sheaves of finite rank just as it takes place in the case of smooth vector bundles.

Therefore, we consider Lie supergroups and principal superbundles in the category of $G$-supermanifolds. Our goal here is the following extension of Theorem 1 to principal $G$-superbundles.

Theorem 2. Let $\widehat{P} \rightarrow \widehat{M}$ be a principal $G$-superbundle with a structure $G$-Lie supergroup $\widehat{K}$, and let $\widehat{H}$ be a closed $G$-Lie supersubgroup of $\widehat{K}$ such that $\widehat{K} \rightarrow \widehat{K} / \widehat{H}$ is a principal superbundle. There is one-to-one correspondence between the principal $G$-supersubbundles of $\widehat{P}$ with the structure $G$-Lie supergroup $\widehat{H}$ and the global sections of the quotient superbundle $\widehat{P} / \widehat{H} \rightarrow \widehat{M}$ with the typical fiber $\widehat{K} / \widehat{H}$.

A key point is that underlying spaces of $G$-supermanifolds are smooth real manifolds, but possessing very particular transition functions. Therefore, the condition of local triviality of the quotient $\widehat{K} \rightarrow \widehat{K} / \widehat{H}$ is rather restrictive. It is satisfied in the case of DeWitt $G$-supermanifolds [23] and in the most interesting case for applications when $\widehat{K}$ is a supermatrix group and $\widehat{H}$ is its Cartan supersubgroup. For instance, let $\widehat{P}=L \widehat{M}$ be a principal superbundle of graded frames in the tangent superspaces over a supermanifold $\widehat{M}$ of evenodd dimensione $(n, 2 m)$. If its structure general linear supergroup $\widehat{K}=\widehat{G L}(n \mid 2 m ; \Lambda)$ is reduced to the orthgonal-symplectic supersubgroup $\widehat{H}=\widehat{O S} p(n \mid m ; \Lambda)$, one can think of 
the corresponding global section of the quotient bundle $L \widehat{M} / \widehat{H} \rightarrow \widehat{M}$ as being a supermetric on $\widehat{M}$.

\section{Supermanifolds}

Let $V$ be a real vector space. Its exterior algebra

$$
\Lambda=\Lambda_{0} \oplus \Lambda_{1}=\wedge V=\mathbb{R} \underset{k=1}{\oplus} \wedge V=\left(\mathbb{R} \underset{k=1}{\oplus} \wedge \wedge^{2 k} V\right) \oplus\left(\underset{k=1}{\oplus} \wedge^{2 k-1} V\right)
$$

is a graded commutative ring of rank $N=\operatorname{dim} V$. We consider Grassmann algebras of this type. A Grassmann algebra admits the splitting

$$
\Lambda=\mathbb{R} \oplus \mathcal{R}=\mathbb{R} \oplus \mathcal{R}_{0} \oplus \mathcal{R}_{1}=\mathbb{R} \oplus\left(\Lambda_{1}\right)^{2} \oplus \Lambda_{1}
$$

where $\mathcal{R}$ is the ideal of nilpotents of $\Lambda$. It is a unique maximal ideal of $\Lambda$, i.e., $\Lambda$ is a local ring. The corresponding projections $\sigma: \Lambda \rightarrow \mathbb{R}$ and $s: \Lambda \rightarrow \mathcal{R}$ are called the body and soul maps, respectively. Given a basis $\left\{c^{i}\right\}$ for the vector space $V$, elements of the Grassmann algebra take the form

$$
a=\sum_{k=0}^{N} \frac{1}{k !} a_{i_{1} \cdots i_{k}} c^{i_{1}} \cdots c^{i_{k}} .
$$

A Grassmann algebra $\Lambda$ is a graded commutative Banach ring with respect to the norm

$$
\|a\|_{\Lambda}=\sum_{k=0}^{N} \sum_{\left(i_{1} \cdots i_{k}\right)} \frac{1}{k !}\left|a_{i_{1} \cdots i_{k}}\right| .
$$

This norm provides $\Lambda$ with the Euclidean topology of a $2^{N}$-dimensional real vector space.

Let $B=B_{0} \oplus B_{1}=\mathbb{R}^{n} \oplus \mathbb{R}^{m}$ be an $(n, m)$-dimensional graded vector space. Given a Grassmann algebra $\Lambda$ of rank $N$, the $\Lambda$-envelope of $B$ is a free graded $\Lambda$-module

$$
B^{n \mid m}=\Lambda B=(\Lambda B)_{0} \oplus(\Lambda B)_{1}=\left(\Lambda_{0}^{n} \oplus \Lambda_{1}^{m}\right) \oplus\left(\Lambda_{1}^{n} \oplus \Lambda_{0}^{m}\right),
$$

of rank $n+m$. It is called the superspace. Its even part

$$
B^{n, m}=\Lambda_{0}^{n} \oplus \Lambda_{1}^{m}
$$

is a $\Lambda_{0}$-module called the $(n, m)$-dimensional supervector space. In accordance with the decomposition (1), any element $q \in B^{n, m}$ is uniquely split as

$$
q=x+y=\left(\sigma\left(x^{i}\right)+s\left(x^{i}\right)\right) e_{i}^{0}+y^{j} e_{j}^{1},
$$


where $\left\{e_{i}^{0}, e_{j}^{1}\right\}$ is a basis for $B$ and $\sigma\left(x^{i}\right) \in \mathbb{R}, s\left(x^{i}\right) \in \mathcal{R}_{0}, y^{j} \in \mathcal{R}_{1}$. The corresponding body and soul maps read

$$
\sigma^{n, m}: B^{n, m} \rightarrow \mathbb{R}^{n}, \quad s^{n, m}: B^{n, m} \rightarrow \mathcal{R}^{n, m}=\mathcal{R}_{0}^{n} \oplus \mathcal{R}_{1}^{m}
$$

A supervector space $B^{n, m}$ is provided with the Euclidean topology of a $2^{N-1}(n+m)$ dimensional real vector space.

The superspace $B^{n \mid m}$ seen as a $\Lambda_{0}$-module is isomorphic to the supervector space $B^{n+m, n+m}$. Any $\Lambda$-module endomorphism of $B^{n \mid m}$ is represented by an $(n+m) \times(n+m)$ matrix

$$
L=\left(\begin{array}{ll}
L_{1} & L_{2} \\
L_{3} & L_{4}
\end{array}\right)
$$

with entries taking values in $\Lambda$. It is called a supermatrix. A supermatrix $L$ is even (resp. odd) if $L_{1}$ and $L_{4}$ have even (resp. odd) entries, while $L_{2}$ and $L_{3}$ have the odd (resp. even) ones. Endowed with this gradation, supermatrices (4) make up a $\Lambda$-graded algebra. A supermatrix $L(4)$ is invertible iff the real matrix $\sigma(L)$ is invertible. Invertible supermatrices constitute a general linear graded group $G L(n \mid m ; \Lambda)$.

Turn now to the notion of a superfunction. Let $B^{n, m}$ be a supervector space (3), where $\Lambda$ is a Grassmann algebra of rank $0<m \leq N$. Let $\Lambda^{\prime}$ be a Grassmann subalgebra of $\Lambda$ of rank $N^{\prime}$ whose basis $\left\{c^{a}\right\}$ is a subset of a basis for $\Lambda$. Furthermore, $\Lambda$ is regarded as a $\Lambda^{\prime}$-algebra. Given an open subset $U \subset \mathbb{R}^{n}$, let us consider a $\Lambda^{\prime}$-valued graded function

$$
f(z)=\sum_{k=0}^{N^{\prime}} \frac{1}{k !} f_{a_{1} \ldots a_{k}}(z) c^{a_{1}} \cdots c^{a_{k}}
$$

on $U$ with smooth real coefficients $f_{a_{1} \cdots a_{k}} \in C^{\infty}(U)$. It is prolonged onto $\left(\sigma^{n, 0}\right)^{-1}(U) \subset B^{n, 0}$ as the Taylor series

$$
f(x)=\sum_{k=0}^{N^{\prime}} \frac{1}{k !}\left[\sum_{p=0}^{N} \frac{1}{p !} \frac{\partial^{q} f_{a_{1} \ldots a_{k}}}{\partial z^{i_{1}} \cdots \partial z^{i_{p}}}(\sigma(x)) s\left(x^{i_{1}}\right) \cdots s\left(x^{i_{p}}\right)\right] c^{a_{1}} \cdots c^{a_{k}} .
$$

Then a superfunction $F(q)=F(x, y)$ on $\left(\sigma^{n, m}\right)^{-1}(U) \subset B^{n, m}$ is defined as the sum

$$
F(x, y)=\sum_{r=0}^{N} \frac{1}{r !} f_{j_{1} \ldots j_{r}}(x) y^{j_{1}} \cdots y^{j_{r}}
$$

where $f_{j_{1} \ldots j_{r}}(x)$ are graded functions (6). The representation of a superfunction $F(x, y)$ by the sum (7) however need not be unique. The germs of superfunctions (7) constitute the sheaf $S_{N^{\prime}}$ of graded commutative $\Lambda^{\prime}$-algebras on $B^{n, m}$. At the same time, one can think of a superfunction $(7)$ as being a smooth map of a $2^{N-1}(n+m)$-dimensional Euclidean space $B^{n, m}$ to the $2^{N}$-dimensional one $\Lambda$. 
Using the representation (7), one can define derivatives of superfunctions as follows. Given a superfunction $f(x)(6)$ on $B^{n, 0}$, its derivative with respect to an even argument $x^{i}$ is defined in a natural way as

$$
\partial_{i} f(x)=\sum_{k=0}^{N^{\prime}} \frac{1}{k !}\left[\sum_{p=0}^{N} \frac{1}{p !} \frac{\partial^{p+1} f_{a_{1} \ldots a_{k}}}{\partial z^{i} \partial z^{i_{1}} \cdots \partial z^{i_{p}}}(\sigma(x)) s\left(x^{i_{1}}\right) \cdots s\left(x^{i_{p}}\right)\right] c^{a_{1}} \cdots c^{a_{k}} .
$$

This even derivative is extended to superfunctions $F$ on $B^{n, m}$ in spite of the fact that the representation (7) is not unique. However, the definition of odd derivatives of superfunctions is more intricate.

Let $S_{N^{\prime}}^{0} \subset S_{N^{\prime}}$ be the subsheaf of superfunctions $F(x, y)=f(x)$ (6) independent of the odd arguments $y^{j}$. Let $\wedge \mathbb{R}^{m}$ be the Grassmann algebra generated by the elements $\left(a^{1}, \ldots, a^{m}\right)$. The expression (7) implies the sheaf epimorphism

$$
\lambda: S_{N^{\prime}}^{0} \otimes \wedge \mathbb{R}^{m} \rightarrow S_{N^{\prime}}, \quad \sum_{r=0}^{m} \frac{1}{r !} f_{j_{1} \ldots j_{r}}(x) \otimes\left(a^{j_{1}} \cdots a^{j_{r}}\right) \rightarrow \sum_{r=0}^{m} \frac{1}{r !} f_{j_{1} \ldots j_{r}}(x) y^{j_{1}} \cdots y^{j_{r}} .
$$

This epimorphism is injective and, consequently, is an isomorphism iff

$$
N-N^{\prime} \geq m
$$

If the condition (9) holds, the representation of any superfunction $F$ by the sum (7) is unique, and $F$ is an image of some section $f_{\iota} \otimes a^{\iota}$ of the sheaf $S_{N^{\prime}}^{0} \otimes \wedge \mathbb{R}^{m}$. Then the odd derivative of $F$ is defined as

$$
\frac{\partial}{\partial y^{j}}\left(\lambda\left(f_{\iota} \otimes a^{\iota}\right)\right)=\lambda\left(f_{\iota} \otimes \frac{\partial}{\partial a^{j}}\left(a^{\iota}\right)\right) .
$$

This definition is consistent only if $\lambda$ is an isomorphism. If otherwise, there exists a nonvanishing element $f_{\iota} \otimes a^{\iota}$ such that $\lambda\left(f_{\iota} \otimes a^{\iota}\right)=0$, but $\lambda\left(f_{\iota} \otimes \partial_{j}\left(a^{\iota}\right)\right) \neq 0$.

With the condition (9), one classifies superfunctions as follows [19, 24].

(i) If the condition (9) is satisfied, superfunctions (7) are called $G H^{\infty}$-superfunctions.

(ii) If $N^{\prime}=0$, the condition (9) holds, and we deal with $H^{\infty}$-superfunctions

$$
F(x, y)=\sum_{r=0}^{m} \frac{1}{r !}\left[\sum_{p=0}^{N} \frac{1}{p !} \frac{\partial^{q} f_{j_{1} \ldots j_{r}}}{\partial z^{i_{1}} \cdots \partial z^{i_{p}}}(\sigma(x)) s\left(x^{i_{1}}\right) \cdots s\left(x^{i_{p}}\right)\right] y^{j_{1}} \cdots y^{j_{r}}
$$

where $f_{j_{1} \ldots j_{r}}$ are smooth real functions.

(iii) If $N^{\prime}=N$, the inequality (9) is not satisfied, unless $m=0$. This is the case of $G^{\infty}$-superfunctions.

Superfunctions of these three types are called smooth superfunctions. They however are effected by serious inconsistencies. Firstly, the odd derivatives of $G^{\infty}$-superfunctions 
are ill defined. Secondly, a space of values of $G H^{\infty}$-superfunctions changes from point to point because a Grassmann algebra $\Lambda$ fails to be a free $\Lambda^{\prime}$-module. Though the $H^{\infty}$ superfunctions are free of these defects, they are rather particular, namely, they are even on $B^{n, 0}$ and real on $\mathbb{R}^{n} \subset B^{n, 0}$. The notion of $G$-superfunctions overcomes these difficulties.

Let $\mathcal{G H}_{N^{\prime}}$ denote the sheaf of $G H^{\infty}$-superfunctions on a supervector space $B^{n, m}$. Let us consider the sheaf of graded commutative $\Lambda$-algebras

$$
\mathcal{G}_{N^{\prime}}=\mathcal{G H}_{N^{\prime}} \underset{\Lambda^{\prime}}{\otimes} \Lambda
$$

There is its evaluation morphism

$$
\delta: \mathcal{G}_{N^{\prime}} \ni F \otimes a \mapsto F a \in C^{0 \Lambda}\left(B^{n, m}\right)
$$

to the ring $C^{0 \Lambda}\left(B^{n, m}\right)$ of continuous $\Lambda$-valued functions on $B^{n, m}$. It is an epimorphism onto the sheaf $\mathcal{G}^{\infty}$ of $G^{\infty}$-superfunctions on $B^{n, m}$. A key point is that, for any two integers $N^{\prime}$ and $N^{\prime \prime}$ satisfying the condition (9), there is the canonical isomorphism of sheaves of graded commutative $\Lambda$-algebras $\mathcal{G}_{N^{\prime}}$ and $\mathcal{G}_{N^{\prime \prime}}$. Therefore, given the sheaf $\mathcal{H}^{\infty}$ of $H^{\infty}$-superfunctions $F(10)$ on a supervector space $B^{n, m}$, it suffices to consider the canonical sheaf

$$
\mathcal{G}_{n, m}=\mathcal{H}^{\infty} \otimes \Lambda, \quad \delta: \mathcal{G}_{n, m} \rightarrow C_{B^{n, m}}^{0 \Lambda}
$$

of graded commutative $\Lambda$-algebras on $B^{n, m}$. Its sections are called $G$-superfunctions.

It is important from the geometric viewpoint that the sheaf $\mathfrak{d} \mathcal{G}_{n, m}$ of graded derivations of the sheaf $\mathcal{G}_{n, m}(11)$ is a locally free sheaf of $\mathcal{G}_{n, m}$-modules of rank $(n, m)$. On any open set $U \subset B^{n, m}$, the $\mathcal{G}_{n, m}(U)$-module $\mathfrak{d} \mathcal{G}_{n, m}(U)$ is generated by the derivations $\partial / \partial x^{i}, \partial / \partial y^{j}$ which act on $\mathcal{G}_{n, m}(U)$ by the rule.

$$
\frac{\partial}{\partial x^{i}}(F \otimes a)=\frac{\partial F}{\partial x^{i}} \otimes a, \quad \frac{\partial}{\partial y^{j}}(F \otimes a)=\frac{\partial F}{\partial y^{j}} \otimes a .
$$

Definition 3. Given two open subsets $U$ and $V$ of a supervector space $B^{n, m}$, a map $\phi: U \rightarrow V$ is called supersmooth if it is a set of $n+m$ smooth superfunctions. A Hausdorff paracompact topological space $M$ is said to be an $(n, m)$-dimensional smooth supermanifold if it admits an atlas

$$
\Psi=\left\{U_{\zeta}, \phi_{\zeta}\right\}, \quad \phi_{\zeta}: U_{\zeta} \rightarrow B^{n, m}
$$

such that the transition functions $\phi_{\zeta} \circ \phi_{\xi}^{-1}$ are supersmooth. If transition functions are

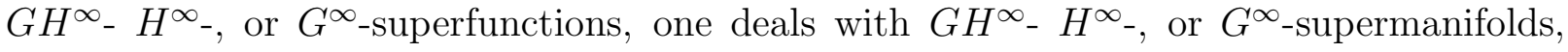
respectively. 
By virtue of Definition 3, any smooth supermanifold of dimension $(n, m)$ also carries a structure of a smooth real manifold of dimension $2^{N-1}(n+m)$, whose atlas however possesses rather particular transition functions. Therefore, it may happen that non-isomorphic smooth supermanifolds are diffeomorphic as smooth manifolds.

Similarly to the case of smooth manifolds, Definition 3 of smooth supermanifolds is equivalent to the following one $[15,19]$.

Definition 4. A smooth supermanifold is a graded local-ringed space $(M, S)$ with an underlying topological space $M$ and the structure sheaf $S$ which is locally isomorphic to the graded local-ringed space $\left(B^{n, m}, \mathcal{S}\right)$, where $\mathcal{S}$ is one of the sheaves of smooth superfunctions on $B^{n, m}$.

By a morphism of smooth supermanifolds is meant their morphism as local-ringed spaces

$$
(\varphi, \Phi):(M, S) \rightarrow\left(M^{\prime}, S^{\prime}\right), \quad \varphi: M \rightarrow M^{\prime}, \quad \Phi\left(S^{\prime}\right)=\left(\varphi_{*} \circ \varphi^{*}\right)\left(S^{\prime}\right) \subset \varphi_{*}(S),
$$

where $\varphi_{*}(S)$ is the direct image of a sheaf and $\varphi^{*} S^{\prime}$ is the inverse (pull-back) one. The condition $\varphi^{*}\left(S^{\prime}\right) \subset S$ implies that $\varphi: M \rightarrow M^{\prime}$ is a smooth map.

The notion of a $G$-supermanifold follows Definition 4 of smooth supermanifolds.

Definition 5. A $G$-supermanifold is a graded local-ringed space $\widehat{M}=\left(M, \mathcal{G}_{M}\right)$ satisfying the following conditions:

(i) $M$ is a Hausdorff paracompact topological space;

(ii) $\left(M, \mathcal{G}_{M}\right)$ is locally isomorphic to the graded local-ringed space $\left(B^{n, m}, \mathcal{G}_{n, m}\right)$, where $\mathcal{G}_{n, m}$ is the sheaf of $G$-superfunctions on $B^{n, m}$;

(iii) there exists an evaluation morphism $\delta: \mathcal{G}_{M} \rightarrow C_{M}^{0 \Lambda}$ to the sheaf $C_{M}^{0 \Lambda}$ of continuous $\Lambda$-valued functions on $M$ which is locally compatible to the evaluation morphism (11).

In particular, the triple $\widehat{B}^{n, m}=\left(B^{n, m}, \mathcal{G}_{n, m}, \delta\right)$, where $\delta$ is the evaluation morphism (11), is called the standard $G$-supermanifold.

Any $G H^{\infty}$-supermanifold $\left(M, G H_{M}^{\infty}\right)$ with the structure sheaf $\mathcal{G H}_{M}^{\infty}$ is naturally extended to the $G$-supermanifold $\left(M, \mathcal{G}_{M}=\mathcal{G H}_{M}^{\infty} \otimes \Lambda\right)$. Every $G$-supermanifold defines the underlying $G^{\infty}$-supermanifold $\left(M, \mathcal{G}_{M}^{\infty}=\delta\left(\mathcal{G}_{M}\right)\right)$. Therefore, the underlying space $M$ of a $G$-supermanifold $\left(M, \mathcal{G}_{M}\right)$ is provided with the structure of a real smooth manifold of dimension $2^{N-1}(n+m)$. However, it may happen that non-isomorphic $G$-supermanifolds have diffeomorphic underlying smooth manifolds.

Given a $G$-supermanifold $\left(M, \mathcal{G}_{M}\right)$, the $\operatorname{ring} \mathcal{G}_{M}(M)$ of $G$-superfunctions on $M$ becomes a Fréchet algebra with respect to the topology of uniform convergence of derivatives of any order defined by the family of seminorms

$$
p_{l, K}(F)=\max _{q \in K,\left(\alpha_{1}+\cdots+\alpha_{k}\right) \leq l}\left\|\delta\left(\left(\frac{\partial}{\partial x^{i_{1}}}\right)^{\alpha_{1}} \cdots\left(\frac{\partial}{\partial x^{i_{k}}}\right)^{\alpha_{k}} \sum_{r=0}^{m} \sum_{\left(j_{1} \ldots j_{r}\right)} \frac{\partial}{\partial y^{j_{1}}} \cdots \frac{\partial}{\partial y^{j_{r}}} F\right)(q)\right\|_{\Lambda},
$$


where $l \in \mathbb{N}$ and $K$ runs over compact subsets of $M$. Given the standard $G$-supermanifold $\left(B^{n, m}, \mathcal{G}_{n, m}\right)$ and an open $U \subset B^{n, m}$, there is an isomertical isomorphism of Fréchet algebras

$$
\mathcal{G}_{n, m}(U) \equiv C^{\infty \Lambda}\left(\sigma^{n, m}(U)\right) \otimes \wedge \mathbb{R}^{m}=C^{\infty}\left(\sigma^{n, m}(U)\right) \otimes \Lambda \otimes \wedge \mathbb{R}^{m}
$$

where $C^{\infty}\left(\mathbb{R}^{m}\right) \otimes \Lambda \otimes \wedge \mathbb{R}^{m}$ is provided with the corresponding topology of uniform convergence of derivatives of any order. As a consequence, the evaluation morphism $\delta$ (11) takes its values into the sheaf $C_{B^{n, m}}^{\infty \Lambda}$ of smooth $\Lambda$-valued functions on $B^{n, m}$. Accordingly, the evaluation morphism of a $G$-supermanifold $\left(M, \mathcal{G}_{M}\right)$ is $\delta: \mathcal{G}_{M} \rightarrow C_{M}^{\infty \Lambda}$.

By a morphism of $G$-supermanifolds is meant their morphism as local-ringed spaces

$$
\widehat{\varphi}=(\varphi, \Phi):\left(M, \mathcal{G}_{M}\right) \rightarrow\left(M^{\prime}, \mathcal{G}_{M^{\prime}}\right)
$$

over the pull-back morphism $\left(\varphi, \varphi_{*} \circ \varphi^{*}\right)$ of the underlying $G^{\infty}$-supermanifolds. It follows that $\varphi: M \rightarrow M^{\prime}$ is a smooth map of underlying smooth manifolds. Note that a map $\varphi$ is not sufficient on its own in order to determine an even sheaf morphism $\Phi$, and additional specification $\Phi$ is needed.

A $G$-morphism (13) is said to be a monomorphism (resp. epimorphism) if $\varphi$ is injective (resp. surjective) and $\Phi$ is an epimorphism (resp. monomorphism). In particular, a $G$ monomorphism $\widehat{\varphi}: \widehat{M}^{\prime} \rightarrow \widehat{M}$ is called a supersubmanifold if a morphism of underlying manifolds $\varphi: M^{\prime} \rightarrow M$ is a submanifold, i.e., an injective immersion. If $\varphi$ is imbedding, a supersubmanifold is called imbedded.

\section{Superbundles}

As was mentioned above, we consider superbundles in the category of $G$-supermanifolds $[15,19]$. Therefore, we start with the definition of a product of two $G$-supermanifolds.

Let $\left(B^{n, m}, \mathcal{G}_{n, m}\right)$ and $\left(B^{r, s}, \mathcal{G}_{r, s}\right)$ be two standard $G$-supermanifolds. Given open sets $U \subset B^{n, m}$ and $V \subset B^{r, s}$, we consider the presheaf

$$
U \times V \rightarrow \mathcal{G}_{n, m}(U) \widehat{\otimes} \mathcal{G}_{r, s}(V)
$$

where $\widehat{\otimes}$ denotes the tensor product of modules completed in the Grothendieck topology. This presheaf yields the structure sheaf $\mathcal{G}_{n+r, m+s}$ of the standard $G$-supermanifold $\widehat{B}^{n+r, m+s}$. This construction is generalized to arbitrary $G$-supermanifolds as follows.

Let $\left(M, \mathcal{G}_{M}\right)$ and $\left(M^{\prime}, \mathcal{G}_{M^{\prime}}\right)$ be two $G$-supermanifolds of dimensions $(n, m)$ and $(r, s)$, respectively. Their product $\left(M, \mathcal{G}_{M}\right) \times\left(M^{\prime}, \mathcal{G}_{M^{\prime}}\right)$ is defined as the graded local-ringed space $\left(M \times M^{\prime}, \mathcal{G}_{M} \widehat{\otimes} \mathcal{G}_{M^{\prime}}\right)$, where $\mathcal{G}_{M} \widehat{\otimes} \mathcal{G}_{M^{\prime}}$ is the sheaf constructed from the presheaf

$$
U \times U^{\prime} \rightarrow \mathcal{G}_{M}(U) \widehat{\otimes} \mathcal{G}_{M^{\prime}}\left(U^{\prime}\right)
$$


for any open subsets $U \subset M$ and $U^{\prime} \subset M^{\prime}$. This product is a $G$-supermanifold of dimension $(n+r, m+s)$ provided with the evaluation morphism

$$
\delta: \mathcal{G}_{M} \widehat{\otimes} \mathcal{G}_{M^{\prime}} \rightarrow C_{M \times M^{\prime}}^{\infty \Lambda}
$$

There are the canonical $G$-epimorphisms

$$
\widehat{\operatorname{pr}}_{1}:\left(M, \mathcal{G}_{M}\right) \times\left(M^{\prime}, \mathcal{G}_{M^{\prime}}\right) \rightarrow\left(M, \mathcal{G}_{M}\right), \quad \widehat{\operatorname{pr}}_{2}:\left(M, \mathcal{G}_{M}\right) \times\left(M^{\prime}, \mathcal{G}_{M^{\prime}}\right) \rightarrow\left(M^{\prime}, \mathcal{G}_{M^{\prime}}\right),
$$

which one can think of as being trivial superbundles.

Definition 6. A (locally trivial) superbundle over a $G$-supermanifold $\widehat{M}=\left(M, \mathcal{G}_{M}\right)$ with a typical fiber $\widehat{F}=\left(F, \mathcal{G}_{F}\right)$ is a pair $(\widehat{Y}, \widehat{\pi})$ of a $G$-supermanifold $\widehat{Y}=\left(Y, \mathcal{G}_{Y}\right)$ and a $G$-epimorphism

$$
\widehat{\pi}:\left(Y, \mathcal{G}_{Y}\right) \rightarrow\left(M, \mathcal{G}_{M}\right)
$$

such that $M$ admits an open cover $\left\{U_{\alpha}\right\}$ together with a family of local $G$-isomorphisms

$$
\widehat{\psi}_{\alpha}:\left(\pi^{-1}\left(U_{\alpha}\right),\left.\mathcal{G}_{Y}\right|_{\pi^{-1}\left(U_{\alpha}\right)}\right) \rightarrow\left(M,\left.\mathcal{G}_{M}\right|_{U_{\alpha}}\right) \times\left(F, \mathcal{G}_{F}\right) .
$$

For any $q \in M$, by $\widehat{Y}_{q}=\widehat{\pi}^{-1}(q)$ is denoted the $G$-supermanifold

$$
\left(\pi^{-1}(q), \mathcal{G}_{q}=\left.\left(\mathcal{G}_{Y} / \mathcal{M}_{q}\right)\right|_{\pi^{-1}(q)}\right)
$$

where $\mathcal{M}_{q}$ is a subsheaf of $\mathcal{G}_{Y}$ whose sections vanish on $\pi^{-1}(q)$. This supermanifold is a fiber of $\widehat{Y}$ over $q \in M$. By a section of the superbundle (15) over an open set $U \subset M$ is meant a $G$-monomorphism $\widehat{s}:\left(U,\left.\mathcal{G}_{M}\right|_{U}\right) \rightarrow \widehat{Y}$ such that $\widehat{\pi} \circ \widehat{s}$ is the identity morphism of $\left(U,\left.\mathcal{G}_{M}\right|_{U}\right)$. Given another supermanifold $\widehat{Y}^{\prime}$ over $\widehat{M}$, a superbundle morphism $\widehat{\varphi}: \widehat{Y} \rightarrow \widehat{Y}^{\prime}$ is a $G$-morphism such that $\widehat{\pi}^{\prime} \circ \widehat{\varphi}=\widehat{\pi}$.

It is readily observed that, by virtue of Definition 6 , the underlying space $Y$ of a superbundle $\widehat{Y}(15)$ is a smooth fiber bundle over $M$ with the typical fiber $F$. A section $\widehat{s}$ of this superbundle defines a section $s$ of the fiber bundle $Y \rightarrow M$, and a superbundle morphism $\widehat{Y} \rightarrow \widehat{Y}^{\prime}$ is given over a smooth bundle morphism $Y \rightarrow Y^{\prime}$ of their underlying spaces.

A superbundle over a $G$-supermanifold $\widehat{M}=\left(M, \mathcal{G}_{M}\right)$ whose typical fiber is a superspace $\left(B^{n \mid m}, \mathcal{G}_{n \mid m}\right)$ (seen as the standard $(n+m, n+m)$-dimensional $G$-supermanifold) is called a supervector bundle. Transition functions $\widehat{\rho}_{\alpha \beta}=\widehat{\psi}_{\alpha} \circ \widehat{\psi}_{\beta}^{-1}$ of a supervector bundle yield sheaf isomorphisms

$$
\Upsilon_{\alpha \beta}:\left.\left.\mathcal{G}_{n \mid m}\right|_{U_{\alpha} \cap U_{\beta}} \rightarrow \mathcal{G}_{n \mid m}\right|_{U_{\alpha} \cap U_{\beta}}
$$

which are described by matrices whose entries are sections of $\left.\mathcal{G}_{n \mid m}\right|_{U_{\alpha} \cap U_{\beta}}$. Their evaluation $\delta\left(\Upsilon_{\alpha \beta}\right)$ are $G L(n \mid m ; \Lambda)$-valued $G^{\infty}$-functions on $U_{\alpha} \cap U_{\beta}$. Sections of a supervector bundle 
constitute a sheaf of locally free graded $\mathcal{G}_{M}$-modules. Conversely, let $S$ be a sheaf of locally free graded $\mathcal{G}_{M}$-modules of rank $(r, s)$ on a $G$-manifold $\widehat{M}$, there exists a supervector bundle over $\widehat{M}$ such that $S$ is isomorphic to the sheaf of its sections.

For instance, the locally free graded sheaf $\mathfrak{d} \mathcal{G}_{M}$ of graded derivations of $\mathcal{G}_{M}$ defines a supervector bundle, called the tangent superbundle $T \widehat{M}$ of a $G$-supermanifold $\widehat{M}$. If $\left(q^{1}, \ldots, q^{m+n}\right)$ and $\left(q^{1}, \ldots, q^{m+n}\right)$ are two coordinate charts on $M$, the Jacobian matrix

$$
\Upsilon_{j}^{i}=\frac{\partial q^{i}}{\partial q^{j}}
$$

(see the prescription (12)) provides the transition function for $T \widehat{M}$. It should be emphasized that the evaluation $\delta\left(\Upsilon_{j}^{i}\right)$ of the Jacobian matrix (18) cannot be written as the Jacobian matrix since odd derivatives of $G^{\infty}$-superfunctions are ill-defined.

Turn now to the notion of a principal superbundle with a structure $G$-Lie supergroup.

Let $\widehat{e}=(e, \Lambda)$ denote a single point with the trivial $(0,0)$-dimensional $G$-supermanifold structure. For any $G$-supermanifold, there are natural identifications $\widehat{e} \times \widehat{M}=\widehat{M} \times \widehat{e}=\widehat{M}$ we refer to in the sequel. A $G$-supermanifold $\widehat{K}=\left(K, \mathcal{G}_{K}\right)$ is said to be a $G$-Lie supergroup if there exist the following $G$-supermanifold morphisms: a multiplication $\widehat{m}: \widehat{K} \times \widehat{K} \rightarrow \widehat{K}$, a unit $\widehat{\varepsilon}: \widehat{e} \rightarrow \widehat{K}$, an inverse $\widehat{k}: \widehat{K} \rightarrow \widehat{K}$, which satisfy

the associativity $\widehat{m} \circ(\mathrm{Id} \times \widehat{m})=\widehat{m} \circ(\widehat{m} \times \mathrm{Id}): \widehat{K} \times \widehat{K} \times \widehat{K} \rightarrow \widehat{K} \times \widehat{K} \rightarrow \widehat{K}$,

the unit property $(\widehat{m} \circ(\widehat{\varepsilon} \times \operatorname{Id}))(\widehat{e} \times \widehat{K})=(\widehat{m} \circ(\operatorname{Id} \times \widehat{\varepsilon}))(\widehat{K} \times \widehat{e})=\operatorname{Id} \widehat{K}$,

the inverse property $\widehat{m} \circ(\widehat{k} \times \operatorname{Id})(\widehat{K} \times \widehat{K})=\widehat{m} \circ(\operatorname{Id} \times \widehat{k})(\widehat{K} \times \widehat{K})=\widehat{\varepsilon}(\widehat{e})$.

Given a point $g \in K$, let us denote by $\widehat{g}: \widehat{e} \rightarrow \widehat{K}$ the $G$-supermanifold morphism whose image in $K$ is $g$. Then one can define the left and right translations of $\widehat{K}$ as the $G$ supermanifold isomorphisms

$$
\widehat{L}_{g}: \widehat{K}=\widehat{e} \times \widehat{K} \stackrel{\widehat{g} \times \operatorname{Id}}{\longrightarrow} \widehat{K} \times \widehat{K} \stackrel{\widehat{m}}{\longrightarrow} \widehat{K}, \quad \widehat{R}_{g}: \widehat{K}=\widehat{K} \times \widehat{e} \stackrel{\operatorname{Id} \times \widehat{g}}{\longrightarrow} \widehat{K} \times \widehat{K} \stackrel{\widehat{m}}{\longrightarrow} \widehat{K} .
$$

Apparently, the underlying smooth manifold $K$ of a $G$-Lie supergroup $\widehat{K}$ is provided with the structure of a real Lie group, called the underlying Lie group. In particular, the transformations of $K$ corresponding to the left and right translations (19) are ordinary left and right multiplications of $K$ by $g$.

For example, the general linear graded group $G L(n \mid m ; \Lambda)$ is endowed with the natural structure of an $H^{\infty}$-supermanifold of dimension $\left(n^{2}+m^{2}, 2 n m\right)$ so that the matrix multiplication is an $H^{\infty}$-morphism. Thus, $G L(n \mid m ; \Lambda)$ is a $H^{\infty}$-Lie supergroup. It is trivially extended to the $G$-Lie supergroup $\widehat{G L}(n \mid m ; \Lambda)$, called the general linear supergroup.

A homomorphism $\widehat{\varphi}: \widehat{K} \rightarrow \widehat{K}^{\prime}$ of $G$-Lie groups is defined as a $G$-supermanifold morphism which obeys the conditions

$$
\widehat{\varphi} \circ \widehat{m}=\widehat{m}^{\prime} \circ(\widehat{\varphi} \times \widehat{\varphi}) . \quad \widehat{\varphi} \circ \widehat{\varepsilon}=\varepsilon^{\prime}, \quad \widehat{\varphi} \circ \widehat{k}=\widehat{k}^{\prime} \circ \widehat{\varphi} .
$$


In particular, an imbedded monomorphism $\widehat{H} \rightarrow \widehat{K}$ of $G$-Lie supergroups is called a $G$-Lie supersubgroup of $\widehat{K}$. Accordingly, the monomorphism of underlying Lie groups $H \rightarrow K$ is a Lie subgroup of $K$.

By a right action of a $G$-Lie supergroup $\widehat{K}$ on a $G$-supermanifold $\widehat{P}$ is meant a $G$ epimorphism $\widehat{\rho}: \widehat{P} \times \widehat{K} \rightarrow \widehat{P}$ such that

$$
\widehat{\rho} \circ(\widehat{\rho} \times \operatorname{Id})=\widehat{\rho} \circ(\operatorname{Id} \times \widehat{m}): \widehat{P} \times \widehat{K} \times \widehat{K} \rightarrow \widehat{P}, \quad \widehat{\rho} \circ(\operatorname{Id} \times \widehat{\varepsilon})(\widehat{P} \times \widehat{e})=\operatorname{Id} \widehat{P} .
$$

Apparently, this action implies a right action $\rho$ of the underlying Lie group $K$ of $\widehat{K}$ on the underlying manifold $P$ of $\widehat{P}$. Similarly, a left action of a $G$-Lie supergroup on a $G$ supermanifold is defined.

For instance, a $G$-Lie supergroup acts on itself by right and left translations (19). The general linear supergroup $\widehat{G L}(n \mid m ; \Lambda)$ acts linearly on the standard supermanifold $\widehat{B}^{n \mid m}$ on the left by the matrix multiplication which is a $G$-morphism.

A quotient of the action of a $G$-Lie supergroup $\widehat{K}$ on a $G$-supermanifold $\widehat{P}$ is a pair $(\widehat{M}, \widehat{\pi})$ of a $G$-supermanifold $\widehat{M}$ and a $G$-supermanifold morphism $\widehat{\pi}: \widehat{P} \rightarrow \widehat{M}$ such that:

(i) there is the equality

$$
\widehat{\pi} \circ \widehat{\rho}=\widehat{\pi} \circ \widehat{p r}_{1}: \widehat{P} \times \widehat{K} \rightarrow \widehat{M},
$$

(ii) for any morphism $\widehat{\varphi}: \widehat{P} \rightarrow \widehat{M}^{\prime}$ such that $\widehat{\varphi} \circ \widehat{\rho}=\widehat{\varphi} \circ \widehat{p r}_{1}$, there is a unique $G$ morphism $\widehat{\gamma}: \widehat{M} \rightarrow \widehat{M}^{\prime}$ with $\widehat{\varphi}=\widehat{\gamma} \circ \widehat{\pi}$. The quotient $(\widehat{M}, \widehat{\pi})$, denoted by $\widehat{P} / \widehat{K}$, need not exists. If it exists, its underlying space is $M=P / K$, and there is a monomorphism of the structure sheaf $\mathcal{G}_{M}$ of $\widehat{M}$ to the direct image $\pi_{*} \mathcal{G}_{P}$. Since the $G$-Lie group $\widehat{K}$ acts trivially on $\widehat{M}$, the range of this monomorphism is a subsheaf of $\pi_{*} \mathcal{G}_{P}$, invariant under the action of $\widehat{K}$. Moreover, there is an isomorphism $\mathcal{G}_{M} \cong\left(\pi_{*} \mathcal{G}_{P}\right)^{\widehat{K}}$ of $\mathcal{G}_{M}$ to the subsheaf of $\widehat{K}$-invariant sections of $\mathcal{G}_{P}$. The latter is generated by sections of $\mathcal{G}_{P}$ on $\pi^{-1}(U), U \subset M$, which have the same image under the morphisms

$$
\widehat{\rho}^{*}:\left.\left.\mathcal{G}_{P}\right|_{\pi^{-1}(U)} \rightarrow\left(\mathcal{G}_{K} \widehat{\otimes} \mathcal{G}_{P}\right)\right|_{(\widehat{\pi} \circ \widehat{\rho})^{-1}(U)}, \quad \widehat{\operatorname{pr}}_{1}^{*}:\left.\left.\mathcal{G}_{P}\right|_{\pi^{-1}(U)} \rightarrow\left(\mathcal{G}_{K} \widehat{\otimes} \mathcal{G}_{P}\right)\right|_{\left(\widehat{\pi}^{\circ} \widehat{p r}_{1}\right)^{-1}(U)} .
$$

Definition 7. A principal superbundle with a structure $G$-Lie supergroup $\widehat{K}$ is defined as a quotient $\widehat{\pi}: \widehat{P} \rightarrow \widehat{P} / \widehat{K}=\widehat{M}$, which is a locally trivial superbundle with the typical fiber $\widehat{G}$ such that there exists an open cover $\left\{U_{\alpha}\right\}$ of $M$ together with $\widehat{G} K$-equivariant trivialization morphisms

$$
\widehat{\psi}_{\alpha}:\left(\pi^{-1}\left(U_{\alpha}\right),\left.\mathcal{G}_{P}\right|_{\pi^{-1}\left(U_{\alpha}\right)}\right) \rightarrow\left(U_{\alpha},\left.\mathcal{G}_{M}\right|_{U_{\alpha}}\right) \times \widehat{K}
$$

where $\widehat{K}$ acts on itself by left translations. 
Note that, in fact, we need only assumption (i) of the definition of a quotient and the condition of local triviality of $\widehat{P}$. Apparently, the underlying smooth bundle $P \rightarrow M$ of a principal superbundle $\widehat{P} \rightarrow \widehat{P} / \widehat{K}$ is a principal smooth bundle with the structure group $K$.

Given a principal superbundle $\widehat{\pi}: \widehat{P} \rightarrow \widehat{M}$ with a structure $G$-Lie supergroup $\widehat{K}$, let $\widehat{V}$ be a $G$-supermanifold provided with a left action $\varrho: \widehat{K} \times \widehat{V} \rightarrow \widehat{V}$ of $\widehat{K}$. Let us consider the right action of $\widehat{K}$ on the product $\widehat{P} \times \widehat{V}$ defined by the morphisms

$$
\widehat{w}: \widehat{P} \times \widehat{V} \times \widehat{K} \stackrel{\operatorname{Id} \times \widehat{\kappa}}{\longrightarrow} \widehat{P} \times \widehat{K} \times \widehat{V} \stackrel{\operatorname{Id} \times \widehat{\Delta} \times \operatorname{Id}}{\longrightarrow} \widehat{P} \times \widehat{K} \times \widehat{K} \times \widehat{V} \stackrel{\widehat{\rho} \times \widehat{\varrho}^{-1}}{\longrightarrow} \widehat{P} \times \widehat{V},
$$

where $\widehat{\kappa}: \widehat{V} \times \widehat{K} \rightarrow \widehat{K} \times \widehat{V}$ is the morphism exchanging the factors, $\widehat{\Delta}: \widehat{K} \times \widehat{K} \rightarrow \widehat{K}$ is the diagonal morphism, and $\widehat{\varrho}^{-1}=\widehat{\varrho} \circ(\widehat{k} \times$ Id $)$. The corresponding action of the underlying Lie group $K$ on the underlying smooth manifold $P \times V$ reads

$$
(p \times v) g=\left(p g \times g^{-1} v\right), \quad p \in P, \quad v \in V, \quad g \in K .
$$

Then one can show that the quotient $(\widehat{P} \times \widehat{V}) / \widehat{K}$ with respect to the action (21) is a superbundle over $\widehat{M}$ with the typical fiber $\widehat{V}$. It is called a superbundle associated to the principal superbundle $\widehat{P}$ because the underlying smooth manifold $(P \times V) / K$ is a fiber bundle over $M$ associated to the principal bundle $P$.

\section{The proof of Theorem 2}

Let

$$
\widehat{\pi}: \widehat{P} \rightarrow \widehat{P} / \widehat{K}
$$

be a principal superbundle with a structure $G$-Lie group $\widehat{K}$. Let $\widehat{i}: \widehat{H} \rightarrow \widehat{K}$ be a closed $G$-Lie supersubgroup of $\widehat{K}$, i.e., $i: H \rightarrow K$ is a closed Lie subgroup of the Lie group $K$. Since $H$ is a closed subgroup of $K$, the latter is an $H$-principal fiber bundle $K \rightarrow K / H$ [1]. However, $K / H$ need not possesses a $G$-supermanifold structure. Let us assume that the action

$$
\widehat{\rho}: \widehat{K} \times \widehat{H} \stackrel{\operatorname{Id} \times \widehat{i}}{\longrightarrow} \widehat{K} \times \widehat{K} \stackrel{\widehat{m}}{\longrightarrow} \widehat{K}
$$

of $\widehat{H}$ on $\widehat{K}$ by right multiplications defines the quotient

$$
\widehat{\zeta}: \widehat{K} \rightarrow \widehat{K} / \widehat{H}
$$

which is a principal superbundle with the structure $G$-Lie supergroup $\widehat{H}$. In this case, the $G$-Lie supergroup $\widehat{K}$ acts on the quotient supermanifold $\widehat{K} / \widehat{H}$ on the left by the law

$$
\widehat{\varrho}: \widehat{K} \times \widehat{K} / \widehat{H}=\widehat{K} \times \widehat{\zeta}(\widehat{K}) \rightarrow(\widehat{\zeta} \circ \widehat{m})(\widehat{K} \times \widehat{K}) .
$$


Given this action of $\widehat{K}$ on $\widehat{K} / \widehat{H}$, we have a $\widehat{P}$-associated superbundle

$$
\widehat{\Sigma}=(\widehat{P} \times \widehat{K} / \widehat{H}) / \widehat{K} \stackrel{\widehat{\pi}_{\Sigma}}{\longrightarrow} \widehat{M}
$$

with the typical fiber $\widehat{K} / \widehat{H}$. Since

$$
\widehat{P} / \widehat{H}=((\widehat{P} \times \widehat{K}) / \widehat{K}) / \widehat{H}=(\widehat{P} \times \widehat{K} / \widehat{H}) / \widehat{K},
$$

the superbundle $\widehat{\Sigma}(23)$ is the quotient $\left(\widehat{P} / \widehat{H}, \widehat{\pi}_{H}\right)$ of $\widehat{P}$ with respect to the right action

$$
\widehat{\rho} \circ(\operatorname{Id} \times \widehat{i}): \widehat{P} \times \widehat{H} \longrightarrow \widehat{P} \times \widehat{K} \longrightarrow \widehat{P}
$$

of the $G$-Lie supergroup $\widehat{H}$. Let us show that this quotient

$$
\widehat{\pi}_{H}: \widehat{P} \rightarrow \widehat{P} / \widehat{H}
$$

is a principal superbundle with the structure supergroup $\widehat{H}$. Note that, by virtue of the well-known theorem [1], the underlying space $P$ of $\widehat{P}$ is an $H$-principal bundle

$$
\pi_{H}: P \rightarrow P / H
$$

Let $\left\{V_{\kappa}, \widehat{\Psi}_{\kappa}\right\}$ be an atlas of trivializations

$$
\widehat{\Psi}_{\kappa}:\left(\zeta^{-1}\left(V_{\kappa}\right),\left.\mathcal{G}_{K}\right|_{\zeta^{-1}\left(V_{\kappa}\right)}\right) \rightarrow\left(V_{\kappa},\left.\mathcal{G}_{K / H}\right|_{V_{\kappa}}\right) \times \widehat{H},
$$

of the $\widehat{H}$-principal bundle $\widehat{K} \rightarrow \widehat{k} / \widehat{H}$, and let $\left\{U_{\alpha}, \widehat{\psi}_{\alpha}\right\}$ be an atlas of trivializations

$$
\widehat{\psi}_{\alpha}:\left(\pi^{-1}\left(U_{\alpha}\right),\left.\mathcal{G}_{P}\right|_{\pi^{-1}\left(U_{\alpha}\right)}\right) \rightarrow\left(U_{\alpha},\left.\mathcal{G}_{M}\right|_{U_{\alpha}}\right) \times \widehat{K}
$$

of the $\widehat{K}$-principal superbundle $\widehat{P} \rightarrow \widehat{M}$. Then we have the $G$-isomorphisms

$$
\begin{aligned}
\widehat{\psi}_{\alpha \kappa}=\left(\operatorname{Id} \times \widehat{\Psi}_{\kappa}\right) & \circ \widehat{\psi}_{\alpha}:\left(\psi_{\alpha}^{-1}\left(U_{\alpha} \times \zeta^{-1}\left(V_{\kappa}\right)\right),\left.\mathcal{G}_{P}\right|_{\psi_{\alpha}^{-1}\left(U_{\alpha} \times \zeta^{-1}\left(V_{\kappa}\right)\right)}\right) \rightarrow \\
\left(U_{\alpha},\left.\mathcal{G}_{M}\right|_{U_{\alpha}}\right) & \times\left(V_{\kappa},\left.\mathcal{G}_{K / H}\right|_{V_{\kappa}}\right) \times \widehat{H}=\left(U_{\alpha} \times V_{\kappa},\left.\left.\mathcal{G}_{M}\right|_{U_{\alpha}} \widehat{\otimes} \mathcal{G}_{G / H}\right|_{V_{\kappa}}\right) \times \widehat{H}
\end{aligned}
$$

For any $U_{\alpha}$, there exists a well-defined morphism

$$
\begin{aligned}
\widehat{\Psi}_{\alpha}: & \left(\pi^{-1}\left(U_{\alpha}\right),\left.\mathcal{G}_{P}\right|_{U_{\alpha}}\right) \rightarrow\left(U_{\alpha} \times G / H,\left.\mathcal{G}_{M}\right|_{U_{\alpha}} \widehat{\otimes} \mathcal{G}_{K / H}\right) \times \widehat{H}= \\
& \left(U_{\alpha},\left.\mathcal{G}_{M}\right|_{U_{\alpha}}\right) \times \widehat{K} / \widehat{H} \times \widehat{H}
\end{aligned}
$$

such that

$$
\left.\widehat{\Psi}_{\alpha}\right|_{\psi_{\alpha}^{-1}\left(U_{\alpha} \times \zeta^{-1}\left(V_{\kappa}\right)\right)}=\widehat{\psi}_{\alpha \kappa} .
$$


Let $\left\{U_{\alpha}, \widehat{\varphi}_{\alpha}\right\}$ be an atlas of trivializations

$$
\widehat{\varphi}_{\alpha}:\left(\pi_{\Sigma}^{-1}\left(U_{\alpha}\right),\left.\mathcal{G}_{\Sigma}\right|_{\pi_{\Sigma}^{-1}\left(U_{\alpha}\right)}\right) \rightarrow\left(U_{\alpha},\left.\mathcal{G}_{M}\right|_{U_{\alpha}}\right) \times \widehat{K} / \widehat{H}
$$

of the $\widehat{P}$-associated superbundle $\widehat{P} / \widehat{H} \rightarrow \widehat{M}$. Then the morphisms

$$
\left(\widehat{\varphi}_{\alpha}^{-1} \times \mathrm{Id}\right) \circ \widehat{\Psi}_{\alpha}:\left(\pi^{-1}\left(U_{\alpha}\right),\left.\mathcal{G}_{P}\right|_{U_{\alpha}}\right) \rightarrow\left(\pi_{\Sigma}^{-1}\left(U_{\alpha}\right),\left.\mathcal{G}_{\Sigma}\right|_{\pi_{\Sigma}^{-1}\left(U_{\alpha}\right)}\right) \times \widehat{H}
$$

make up an atlas

$$
\left\{\pi_{\Sigma}^{-1}\left(U_{\alpha}\right),\left(\widehat{\varphi}_{\alpha}^{-1} \times \mathrm{Id}\right) \circ \widehat{\Psi}_{\alpha}\right\}
$$

of trivializations of the $\widehat{H}$-principal superbundle $\widehat{P} \rightarrow \widehat{P} / \widehat{H}$.

Now, let

$$
\widehat{i}_{h}: \widehat{P}_{h} \rightarrow \widehat{P}
$$

be an $\widehat{H}$-principal supersubbundle of the principal superbundle $\widehat{P} \rightarrow \widehat{M}$. Then there exists a global section $\widehat{h}$ of the superbundle $\widehat{\Sigma} \rightarrow \widehat{M}$ such that the image of $\widehat{P}_{h}$ with respect to the morphism $\widehat{\pi}_{H} \circ \widehat{i}_{h}$ coincides with the range of the section $\widehat{h}$. Conversely, given a global section $\widehat{h}$ of the superbundle $\widehat{\Sigma} \rightarrow \widehat{M}$, the inverse image $\widehat{\pi}_{H}^{-1}(\widehat{h}(\widehat{M}))$ is an $\widehat{H}$-principal supersubbundle of $\widehat{P} \rightarrow \widehat{M}$.

\section{Supermetrics}

Let us show that, as was mentioned above, the condition of Theorem 2 holds if $\widehat{H}$ is the Cartan supersubgroup of a supermatrix group $\widehat{K}$, i.e., $\widehat{K}$ is a $G$-Lie supersubgroup of some general linear supergroup $\widehat{G L}(n \mid m ; \Lambda)$.

Recall that a Lie superalgebra $\widehat{\mathfrak{g}}$ of an $(n, m)$-dimensional $G$-Lie supergroup $\widehat{K}$ is defined as a $\Lambda$-algebra of left-invariant supervector fields on $\widehat{K}$, i.e., derivations of its structure sheaf $\mathcal{G}_{K}$. A supervector field $u$ is called left-invariant if

$$
(\mathrm{Id} \otimes u) \circ \widehat{m}^{*}=\widehat{m}^{*} \circ u .
$$

Left-invariant supervector fields on $\widehat{K}$ make up a Lie $\Lambda$-superalgebra with respect to the graded Lie bracket

$$
\left[u, u^{\prime}\right]=u \circ u^{\prime}-(-1)^{[u]\left[u^{\prime}\right]} u^{\prime} \circ u,
$$

where the symbol [.] stands for the Grassmann parity. Being a superspace $B^{n \mid m}$, a Lie superalgebra is provided with a structure of the standard $G$-supermanifold $\widehat{B}^{n+m, n+m}$. Its even part $\widehat{\mathfrak{g}}_{0}=\widehat{B}^{n, m}$ is a Lie $\Lambda_{0}$-algebra. 
Let $\widehat{K}$ be a matrix $G$-Lie supergroup. Then there is an exponential map

$$
\xi(J)=\exp (J)=\sum_{k} \frac{1}{k^{\prime}} J^{k}
$$

of some open neighbourhood of the origin of the Lie algebra $\widehat{\mathfrak{g}}_{0}$ onto an open neighbourhood $U$ of the unit of $\widehat{K}$. This map is an $H^{\infty}$-morphism, which is trivially extended to a $G$ morphism.

Let $\widehat{H}$ be a Cartan supersubgroup of $\widehat{K}$, i.e., the even part $\widehat{\mathfrak{h}}_{0}$ of the Lie superalgebra $\widehat{\mathfrak{h}}$ of $w h H$ is a Cartan subalgebra of the Lie algebra $\widehat{\mathfrak{g}}_{0}$, i.e.,

$$
\widehat{\mathfrak{g}}_{0}=\widehat{\mathfrak{f}}_{0}+\widehat{\mathfrak{h}}_{0}, \quad\left[\widehat{\mathfrak{f}}_{0}, \widehat{\mathfrak{f}}_{0}\right] \subset \widehat{\mathfrak{h}}_{0}, \quad\left[\widehat{\mathfrak{f}}_{0}, \widehat{\mathfrak{h}}_{0}\right] \subset \widehat{\mathfrak{f}}_{0} .
$$

Then there exists an open neighbourhood, say again $\widehat{U}$, of the unit of $\widehat{K}$ such that any element $g$ of $\widehat{U}$ is uniquely brought into the form

$$
g=\exp (F) \exp (I), \quad F \in \widehat{\mathfrak{f}}_{0}, \quad I \in \widehat{\mathfrak{h}}_{0} .
$$

Then the open set $\widehat{U}_{H}=\widehat{m}(\widehat{U} \times \widehat{H})$ is $G$-isomorphic to the direct product $\xi\left(\xi^{-1}(U) \cap \widehat{\mathfrak{f}}_{0}\right) \times \widehat{H}$. This product provides a trivialization of an open neighbourhood of the unit of $\widehat{K}$. Acting on this trivialization by left translations $\widehat{L}_{g}, g \in \widehat{K}$, one obtains an atlas of a principal superbundle $\widehat{K} \rightarrow \widehat{H}$.

For instance, let us consider a superspace $B^{n \mid 2 m}$, coordinated by $\left(x^{a}, y^{i}, \bar{y}^{i}\right)$, and the general linear supergroup $\widehat{G L}(n \mid 2 m ; \Lambda)$ of its automorphisms. Let $B^{n \mid 2 m}$ be provided with the $\Lambda$-valued bilinear form

$$
\omega=\sum_{i=1}^{n}\left(x^{i} x^{i}\right)+\sum_{j=1}^{m}\left(y^{j} \bar{y}^{\prime j}-\bar{y}^{j} y^{\prime j}\right)
$$

The supermatrices (4) preserving this bilinear form make up the orthogonal-symplectic supergroup $\widehat{O S} p(n \mid m ; \Lambda)[25]$. It is a Cartan subgroup of $\widehat{G L}(n \mid 2 m ; \Lambda)$. Then one can think of the quotient

$$
\widehat{G L}(n \mid 2 m ; \Lambda) / \widehat{O S} p(n \mid m ; \Lambda)
$$

as being a supermanifold of $\Lambda$-valued bilinear forms on $B^{n \mid 2 m}$ which are brought into the form (25) by general linear supertransformations.

Let $\widehat{M}$ be $G$-supermanifold of dimension $(n, 2 m)$ and $T \widehat{M}$ its tangent superbundle. Let $L \widehat{M}$ be an associated principal superbundle. Let us assume that its structure supergroup $\widehat{G L}(n \mid 2 m ; \Lambda)$ is reduced to the supersubgroup $\widehat{O S} p(n \mid m ; \Lambda)$. Then by virtue of Theorem 2 , there exists a global section $h$ of the quotient

$$
L \widehat{M} / \widehat{O S} p(n \mid m ; \Lambda) \rightarrow \widehat{M}
$$


which can be regarded as a supermetric on a supermanifold $\widehat{M}$.

Note that, bearing in mind physical applications, one can treat the bilinear form (25) as sui generis superextension of the Euclidean metric on the body $\mathbb{R}^{n}=\sigma\left(B^{n \mid m}\right)$ of the superspace $B^{n \mid m}$. However, the body of a supermanifold is ill-defined in general $[9,26]$.

\section{References}

[1] N.Steenrod, The Topology of Fibre Bundles (Princeton Univ. Press, Princeton, 1972).

[2] G.Sardanashvily, On the geometry of spontaneous symmetry breaking, J. Math. Phys. 33 (1992) 1546.

[3] G.Sardanashvily, Geometry of classical Higgs fields, Int. J. Geom. Methods Mod. Phys. 3 (2006) 139-148; arXiv: hep-th/0510168.

[4] D.Ivanenko and G.Sardanashvily, The gauge treatment of gravity, Phys. Rep. 94 (1983) $1-45$.

[5] G.Sardanashvily, Classical gauge theory of gravity, Theor. Math. Phys. 132 (2002) 1163-1171; arXiv: gr-qc/0208054.

[6] P.West, Introduction to Supersymmetry and Supergravity (Singapore, World Scientific, 1990).

[7] J.Wess and J.Bagger, 1992 Supersymmetry and Supergravity, Princeton Series in Physics (Princeton, Princeton Univ. Press, 1992).

[8] P.Binétry, G.Girardi and R.Grimm, Supergravity couplings: A geometric formulation, Phys. Rep. 343 (2001) 255.

[9] D.Ivanenko and G.Sardanashvily, Goldstone type (non-Poincaré) supergravity, Progr. Theor. Phys. 26 (1986) 969.

[10] P.Deligne and J.Morgan, Notes on supersymmetry (following Joseph Bernstein), In: Quantum Field Theory and Strings: a Course for Mathematicians (Providence, RI: Amer. Math. Soc., 1999) p.41.

[11] C.Carmeli, G.Cassinelli, A.Toigo and V.Varadarajan, Unitary representations of super Lie groups and applications to the classification and multiplet structure of super particles, Commun. Math. Phys. 263 (2006) 217.

[12] A.Almorox, Supergauge theories in graded manifolds, In: Differential Geometric Methods in Mathematical Physics, Lect. Notes in Math. 1251 (Berlin, Springer, 1987) p.114 
[13] C.Boyer and A.Sanchez Valenzuela, Lie supergroup actions on supermanifolds, Trans. Am. Math. Soc. 323 (1991) 151.

[14] T.Stavracou, Theory of connections on graded principal bundles, Rev. Math. Phys. 10 (1998) 47.

[15] L.Mangiarotti and G.Sardanashvily, Connections in Classical and Quantum Field Theory (World Scientific, Singapore, 2000).

[16] M.Zirnbauer, Riemannian symmetric superspaces and their origin in random-matrix theory, J. Math. Phys. 37 (1996) 4986.

[17] D.Alekseevsky, V.Cortés, C.Devchand and U.Semmelman, Killing spinors are Killing vectors in Riemannian supergeometry, J. Geom. Phys. 26 (1998) 37.

[18] O.Goertsches, Riemannian supergeometry, arXiv math.DG/0604143.

[19] C.Bartocci, U.Bruzzo and D.Hernández Ruipérez, The Geometry of Supermanifolds (Dordrecht, Kluwer Academic Publ.,1991).

[20] M.Rothstein, The axioms of supermanifolds and a new structure arising from them, Trans. Amer. Math. Soc. 297 (1986) 159.

[21] C.Bartocci, U.Bruzzo, D.Hernández Ruipérez and V.Pestov, Foundations of supermanifold theory: the axiomatic approach, Diff. Geom. Appl. 3 (1993) 135.

[22] U.Bruzzo and V.Pestov, On the structure of DeWitt supermanifolds, J. Geom. Phys. 30 (1999) 147.

[23] A.Kleppe and C.Wainwright, Supercost space geometry, J. Math. Phys. 48 (2007) 053511.

[24] A.Rogers, Graded manifolds, supermanifolds and infinite-dimensional Grassmann algebras, Commun. Math. Phys. 105 (1986) 275.

[25] D.Fuks, Cohomology of Infinite-Dimensional Lie Algebras (Consultants Bureau, N.Y., 1986).

[26] R.Catenacci, C.Reina and P.Teoflatto, On the body of supermanifolds, J. Math. Phys. 26 (1985) 671. 